[Agr. Biol. Chem., Vol. 29, No. 5, p. 488 489, 1965]

\title{
cis-2-Penten-1-ol in the Essential Oil from Freshly Plucked Tea-Leaves and Black Tea
}

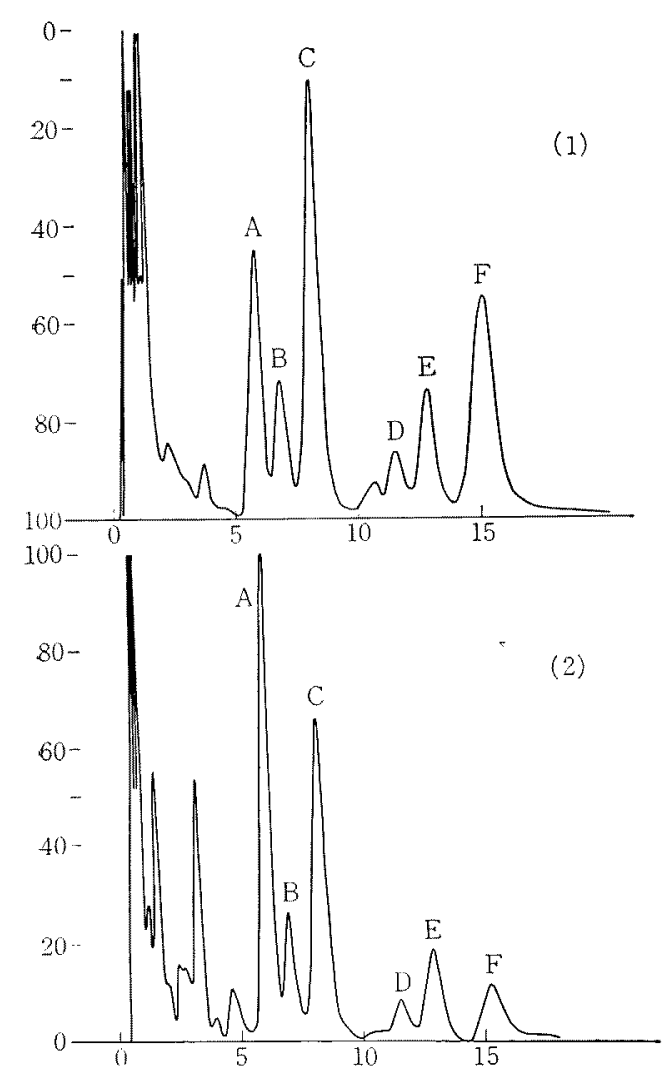

FIG. 1. Gas Chromatograms of the Neutral Fraction (ester and carbonyl comps. free) of the Essential Oil $\left(150^{\circ} \mathrm{C}, 35 \mathrm{ml} / \mathrm{min}\right.$.)

(1) Fresh Tea Leaves

(2) Black Tea

Sir;

The essential oil was extracted from freshly plucked tea-leaves (Benihomare; variety for black tea) and black tea by the same method as reported in the previous paper on green tea flavor. ${ }^{13}$ The gas chromatogram of the 1) T. Yamanishi, T. Kiribuchi, M. Sakai, N. Fujita, Y; Ikeda and K. Sasa, This Journal, 27, 193 (1963). neutral fraction showed twenty-eight distinct peaks among which twelve peaks have been identified with those of known compounds. However, peak A in Fig. 1 was remained unknown and this compound is a component of the essential oil of green tea also. ${ }^{2}$

From the neutral fraction of the essential oil carbonyl compounds were separated by Girard-T reagent and esters were hydrolysed with potassium hydroxide in ethyleneglycol. The carbonyl- and ester-free neutral oil thus obtained was gas chromatographed on a $4 \mathrm{~mm}$ $\times 2 \mathrm{~m}$ column containing 23\% PEG 6000 (polyethyleneglycol) on Shimalite W (celite). The operation was conducted at $150^{\circ} \mathrm{C}$ and $100^{\circ} \mathrm{C}$ with a helium flow rate at $35 \mathrm{ml} / \mathrm{min}$. Relative retention time (Linalool $=1.00$ ) of peak $\mathrm{A}$ was 0.40 , which was a little smaller than those of $n$-hexanol and cis-3-hexen-1-ol (peak $B$ and $\mathrm{C}$ in Fig. 1). The effluent of peak A was trapped under the condition of $100^{\circ} \mathrm{C}$, $35 \mathrm{ml} / \mathrm{min}$ and subjected to IR examination. The spectrum showed strong absorption bands at 3460,1655 and $1320 \mathrm{~cm}^{-1}$. The hydrogenation in the presence of platinum oxide (Adams catalyst) produced $n$-amylalcohol which was identified with the authentic sample by gas chromatography (GLC). From IR data the double bond is cis-form (1655 and $855 \mathrm{~cm}^{-1}$ ) and the shift of $\nu_{c-0-}$ to lower frequency $\left(1038 \mathrm{~cm}^{-1}\right)$ suggests that the constituent of peak $\mathrm{A}$ is cis-2-penten-1-ol, which was identified by GLC and IR with the authentic sample synthesized from propargyl alcohol and ethylbromide through catalytic reduction of 2-pentyn-1-ol (Fig. 2). To certify this constitution trans-2- and trans-3-penten-1-ol were also synthesized, however, their relative

2) T. Yamanishi, T. Kiribuchi, Y. Mikumo, H. Sato and A. Ohmura, This Journal, 29, 300 (1965). 


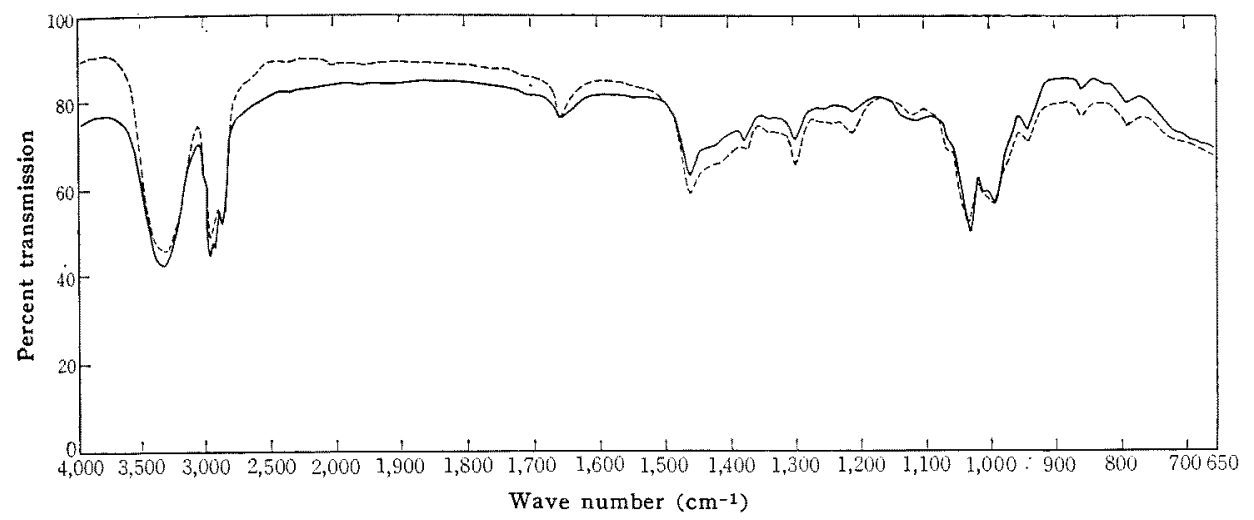

FIG. 2. Infrared Spectra of Natural and Synthetic cis-2-Penten-1-ol.

Full line; natural

Broken line; synthetic (neat film)

retention times in GLC were completely different from that of peak $A$ and their IR spectra showed strong absorption band at $965 \mathrm{~cm}^{-1}$

The presence of cis-2-penten-1-ol has not been reported as a natural product and this compound has a citrous-like fruity flavor and seems to be one of the important constituents of tea flavor.

\section{Akio Kobayashi \\ Hisako Sato \\ Tei Yamanishi}

Laboratory of Food Chemistry Ochanomizu University

Bunkyo-ku, Tokyo 